\title{
Essai d'une géographie symbolique de la morale
}

\author{
Jean-Yves Heurtebise
}

\section{OpenEdition \\ Journals}

\section{Édition électronique}

URL : https://journals.openedition.org/leportique/1533

DOI : 10.4000/leportique.1533

ISSN : $1777-5280$

\section{Éditeur}

Association "Les Amis du Portique"

\section{Référence électronique}

Jean-Yves Heurtebise, «Essai d'une géographie symbolique de la morale », Le Portique [En ligne], ePortique, mis en ligne le 21 décembre 2007, consulté le 11 septembre 2022. URL : http:// journals.openedition.org/leportique/1533; DOI : https://doi.org/10.4000/leportique.1533

Ce document a été généré automatiquement le 11 septembre 2022.

Tous droits réservés 


\title{
Essai d'une géographie symbolique de la morale
}

\author{
Jean-Yves Heurtebise
}

" Je pense à toi Myrtho divine enchanteresse, ... À ton front inondé des clartés d'Orient [...] Et de cendres soudain l'horizon s'est couvert.

Depuis qu'un duc normand brisa tes dieux

d'argile... ${ }^{1}$ "

1 Selon Nietzsche, la naissance de la morale, c'est-à-dire le renversement des valeurs de vie par les idéaux de mort, s'introduit en philosophie par la doctrine parménidienne de l'Être ${ }^{2}$ et la philosophie platonicienne des Idées et s'inscrit dans l'histoire de nos civilisations via l'extension du monothéisme juif puis chrétien ${ }^{3}$. Nous suivrons ici le sens que les concepts de vie et de mort nous semblent avoir chez Nietzsche : par " vie ", nous entendrons donc la faculté plastique de s'approprier le différent et de devenir soimême autre par cette appropriation ; la mort désignant au contraire l'absence de toute faculté de transformation et de renouvellement.

2 Nous voudrions ici changer les termes de ce renversement des valeurs pour lui donner une autre extension et un autre contenu. Nous ferons ainsi l'hypothèse qu'un tel " renversement des valeurs» de vie, d'une part, daterait moins du christianisme qu'il proviendrait du passage historique du Néolithique à l'âge du Bronze et, d'autre part, qu'il se caractériserait par le processus par lequel les valeurs vitalistes, "dionysiennes » et "féminines ", naturalistes et mystiques, esthétiques et contemplatives du «Sud » et de "l'Est » sont peu à peu étouffées par les valeurs ontologiques, " apolliniennes » et « masculines ", égoïstes et militaires, ascétiques et volontaires $d u$ "Nord » et de "l'Ouest » :

«Lorsque Lord Elgin disloqua l'architrave du Parthénon pour emporter plus aisément la frise et les métopes, ça n'était pas pour encastrer ces débris au sein d'un nouvel édifice. Ils finirent à Londres dans les salles glacées d'un temple de la connaissance [...] Ainsi comme si on l'était passé de la prodigalité à l'avarice, l'héritage de la Méditerranée classique fut précautionneusement emmagasiné [...] Le discours des anciens n'incitait plus à l'humanisme libertin et tendre. Il exhortait à la vertu. Il parlait d'obéissance, d'ordre imposé [...] rupture qui, d'un 
coup, au temps des Lumières, retrancha le legs méditerranéen des mouvements du monde ${ }^{4}$.»

La coupure historique de l'âge des métaux

3 Le point de rebroussement historique et géographique, nous le situerons dans le passage du Néolithique, naissance des cités, de la domestication et de l'agriculture ${ }^{5}$, à l'âge du Bronze, naissance des Empires, de l'économie et de la guerre ${ }^{6}$. Sans vouloir entrer dans la sombre querelle des indo-européens, il semblerait que cet aspect guerrier de l'âge du Bronze coïncide avec l'entrée en scène d'un groupe de populations ${ }^{7}$ dont l'unité au moins linguistique apparaît dans certains termes désignant justement : la roue (*rotho-), l'essieu (*hakhs-), le joug et le harnais (*iuk'om), le cheval (*ekhos) (par exemple, pour la roue ou le chariot: ratha- en sanskrit, rota- en latin et rad en allemand) ${ }^{8}$; le métal - fer, cuivre ou bronze : latin aes, gotique aiz, sanskrit ayah et avestique ayo. On peut s'appuyer ici sur un état de la question récent mis au point par Gérard Fussman :

«Les futurs Indo-Aryens ont pénétré en Inde par le Nord-Ouest du sous-continent »; 2. « leurs ancêtres habitaient à l'Ouest de l'Oural et au Nord de la mer Noire et de la Caspienne » : « il faut situer l'habitat des anciens Indo-Iraniens dans la steppe russe ». 3. Ils ont pénétré dans un territoire qui était celui de la civilisation de l'Oxus (2500 à 1500) ; 4. Ce sont des éleveurs nomades : «On peut imaginer les Indo-Aryens voyageant ainsi, poussant leurs troupeaux vers le sud à la recherche de meilleur pâturages, atteignant le piémont nord de l'Hindou-Kouch, le franchissant et pénétrant ensuite par de petites groupes dans le Béloutchistan, le Sind et le Panjab dont les grandes cités (Harappa, Mohen-Daro) étaient déjà dévastées. Le pays n'était pas vide pourtant [...] Mais il y avait de la place pour des éleveurs, et des populations sans habitat fixe, habiles à monter à cheval ou à conduire un char, n'ont jamais eu grand mal à soumettre à leur pouvoir, en fait à piller, des villageois que ne protège pas un fort pouvoir central ${ }^{9}$. "

Quoiqu'il en soit de l'hypothèse indoeuropéenne, on tentera ici de formuler quelques hypothèses dans la mesure où la définition de la révolution néolithique comme simple passage d'une société de cueilleur/chasseur à une société d'agriculteur/éleveur ne semble pas tout à fait satisfaisante. En effet, le cueilleur n'est pas forcément le même que le chasseur, ni l'agriculteur le même que l'éleveur (il faut distinguer la domestication de type agraire qui porte sur des espèces comme le porc, le canard, la poule... et la domestication de type nomade qui porte sur le cheval, le chameau.... ${ }^{10}$. À chaque groupe semble correspondre une certaine distribution géographique et une certaine évolution historique : le cueilleur se base sur le pourtour méditerranéen; le chasseur avance en position plus septentrionale. Or, à un certain moment, les populations de chasseurs - domestiquant le cheval, inventant le fer, la charrue, la courroie, etc. - commencent à se déplacer et à pénétrer dans l'espace des états agraires, lesquels se ferment pour se défendre et, succombant ou non, se constituent alors en empires $^{11}$ (à la formation d'états, par cessation de terres s'opposant aux dons primitifs de biens ${ }^{12}$, succède la formation d'empires et une religion du chef ${ }^{13}$ ). Dès lors le régime de vie collective change : la disposition d'abord aléatoire des rues prend une forme géométrique et hiérarchisée, avec la création d'un centre urbain, politique et religieux qui canalise tous les trajets, et l'élaboration d'une enceinte défensive ${ }^{14}$; à une structure sociale encore relativement horizontale et égalitaire succède une structure sociale verticale et hiérarchisée (castes, ordres...) ${ }^{15}$. L'on serait passer ainsi d'un monde «méditerranéen» focalisé sur la production agricole à un monde "nordique» 
tournant autour de la production technique et des avancées technologiques permettant la victoire dans la guerre.

Relecture de la classification indoeuropéenne et système méridional

Il y aurait un autre élément à ajouter à ce tableau, car ceci nous conduirait à penser que, dans la classification de Dumézil du monde indoeuropéen en trois fonctions: pouvoir, guerre, fécondité ${ }^{16}$, la dernière fonction de fécondité pourrait être un emprunt, pris au dehors :

«La coupure initiale qui sépare les représentants des deux premières fonctions [royauté magique et force brutale] et ceux de la troisième [fécondité] est une donnée indoeuropéenne commune: avec le même développement mythique (séparation initiale; puis indissoluble union dans la structure tripartite hiérarchisée), elle se trouve non seulement à Rome, sur le plan humain, dans le récit des origines de la Ville, guerre sabine et synécisme), mais dans l'Inde, où il est dit que les dieux canoniques du troisième niveau, les Aśvin, n'ont pas d'abord été des dieux et qu'ils ne sont entrés dans la société divine, comme troisième terme, au-dessous des "deux forces", qu'à la suite d'un conflit violent, suivi d'une réconciliation, d'un pacte ${ }^{17}$. "

Si la mythologie est une transcription allégorique de l'histoire, comme le voulait Dumézil, alors l'épisode des Sabines ${ }^{18}$, qui reprend le mythe grec du rapt de Proserpine ${ }^{19}$, traduit la prise de possession violente et guerrière, dans un milieu extérieur au monde indo-européen, des puissances de production et de reproduction naturelles. Autrement dit, la fonction de fécondité ne serait pas "spécifiquement" indoeuropéenne. S'il y a bien, cependant, une troisième fonction qui semble plus «indoeuropéenne ", ce serait plutôt la fonction technique (forgeron et gardien du trésor); ce qui donnerait alors la triade : Zeus, Arès, Héphaïstos. À cet égard, le fait que, dans la mythologie grecque, Aphrodite circule entre Héphaïstos et Arès reflète au plus près cette " réalité » d'une saisie violente du principe féminin par la fonction militaire et de son rattachement à la troisième fonction indoeuropéenne de l'artisanat.

Le féminin de l'histoire et sa géographie

8 Dès lors, le féminin deviendrait, si cette hypothèse est recevable en quelque façon, curieusement absent du monde indoeuropéen. À ce titre, les travaux de Luce Irigaray ${ }^{20}$ et la vieille thèse de Bachofen sur le matriarcat primitif ${ }^{21}$ se rencontrent et nous permettent d'émettre l'hypothèse d'un basculement historique d'une civilisation plutôt "féminine » (agraire, matriarcale...) à une civilisation nettement plus «masculine » (patriarcale, guerrière...).

Cependant, il faut ajouter un très important correctif à cette vision symbolique de l'histoire dans la mesure où elle amène à penser la femme systématiquement sous la figure de la Mère; or avant la femme Mère comme symbole tellurique et producteur, il y a la femme Fée comme symbole cosmique et régénérateur: ce qui est exclu, c'est moins la figure maternelle que la figure "nymphique" de la femme. Cette exclusion semble apparaître dans des cas multiples et fort divers. Ainsi l'Apsara, qui était divinité féminine bénéfique dans l'Inde néolithique, devient démon tentateur avec l'hindouisme ${ }^{22}$. De même encore: Nyi Pahoci, les fées indonésiennes deviendront monstres ${ }^{23}$, les Nymphes des cours d'eau deviendront avec le christianisme Sorcières près des mares ${ }^{24}$ et les Sirènes, détentrices d'un Savoir vénéré, apparaîtront comme de dangereuses ensorceleuses ${ }^{25}$.

Or, ce qui nous surprend dans cette évolution historique et symbolique, dans ce déni progressif de l'aspect féminin de la vie, c'est qu'elle semble être plus ou moins concomitante du mouvement géographique global par lequel le centre d'activité du monde s'est déplacé du Sud vers le Nord (Afrique, Moyen-Orient, Égypte, Grèce, Rome, 
Italie, Espagne, France, Hollande, Angleterre, Amérique ${ }^{26}$, en même temps que de l'Est à l'Ouest. De sorte que, par un profond renversement des valeurs, le Nord (Ouest) barbare est devenu le vecteur de civilisation mondial devant lequel le Sud (Est) doit (devait ?) désormais s'incliner. Ainsi, à la triade masculine et septentrionale du pouvoir, de la guerre et de la production technique (Zeus, Arès, Héphaïstos) s'oppose le couple féminin et méridional de Dionysos et de Cybèle, de la libération cosmique et de la production tellurique, couple de valeurs peu à peu écarté des référents majeurs de la vie et de la pensée humaine.

Religions de la vie, religions de la mort

Pour revenir à la protohistoire, on pourrait maintenant ajouter que, du Néolithique à l'âge du Bronze, il n'y a pas simplement transition de l'agriculture à la guerre mais aussi passage d'une religion qui était d'abord celle des puissances élémentaires (féminines) et des divinités de la fécondité (maternelles) ${ }^{27}$ à une religion des divinités solaires (astrales), de la séparation entre l'âme et le corps ${ }^{28}$ et des juges infernaux (souterrains).

De cet événement témoignerait tout un ensemble de faits.

13 Ainsi, en Égypte, au début de la ve Dynastie, vers 2640 , le Pharaon ne se réclame plus seulement d'Horus, l'animal totémique, mais de Râ, le dieu solaire ${ }^{29}$. D'autre part, l'introduction radicale du culte solaire par Aménophis IV coïncide avec l'apparition de la théorie $\mathrm{du}$ « kou » ou double immatériel du corps. On pourrait tenter de dégager trois strates bien différentes dans Le Livre des morts. Isis et Osiris étaient d'abord des dieux de la fécondité : le démembrement et la recomposition du Dieu illustrant à la fois le rythme de la nature et les mutations du mystique ${ }^{30}$; puis Osiris et Isis devinrent des divinités astrales et la morale constitua l'horizon de l'existence ${ }^{31}$; enfin, ils deviennent les juges des âmes qui, séparées de leur corps, errent dans l'arrière monde souterrain avant de rejoindre, si elles sont sauvées, le monde des astres ${ }^{32}$.

On remarque le même processus global dans la religion romaine, où l'introduction du culte astral et solaire, sous Auguste, coïncide avec un déplacement de la fonction des divinités chtoniennes en divinités infernales ${ }^{33}$. C'est ainsi, par ailleurs, que l'ange gardien, synonyme primitif de la vie, devient symbole théologique de mort: l'âme se manifeste d'abord dans une ombre qui est puissance de vie et de génération avant de devenir réalité dangereuse et mauvaise ${ }^{34}$.

Pour le domaine grec, cette triple évolution se retrouve : du principe homérique de l'Océan vital comme père des êtres et des dieux ${ }^{35}$, on passe au poème hésiodique où Zeus solaire est placé au sommet d'une hiérarchie divine ${ }^{36}$ et, enfin, on aboutit à une nouvelle religion centrée autour des demi-dieux Orphée et Prométhée... qui atteste d'un nouveau rapport de l'homme à la mort. De fait, après sa traversée de l'enfer, Orphée délaisse Dionysos pour adorer Apollon :

« Après qu'il fut descendu dans l'Hadès pour sa femme, et qu'il eut vu les choses d'en bas, Orphée cessa d'honorer Dionysos, alors qu'il considéra Hélios comme le plus grand des dieux, et qu'il lui donna aussi le nom d'Apollon ${ }^{37}$. "

L'intrusion d'une mythologie astrale et psychique à l'intérieur d'une mythologie vitale et naturaliste a donc pour conséquence que le culte de la fécondité devient un culte des morts et que les divinités chtoniennes sont devenues des divinités infernales et juges de la mort. On passe ainsi d'une religion de vie symbolisée par Dionysos ${ }^{38}$ comme dieu des forces cosmiques élémentaires et par Déméter comme symbole de la puissance génésique de la Terre $^{39}$ à une religion de la Mort, introduite par le culte de l'Apollon solaire. Là où Dionysos était affirmation de la vie dans la souffrance et visage rieur même dans le 
$\mathrm{cri}^{40}$, une religion timorée se met en place qui voit dans le mort un refuge où panser ses plaies ${ }^{41}$. Cette nouvelle religion est une religion du Jugement - Perséphone, anciennement fille de Déméter et déesse du printemps, y devient juge greffière de l'enfer auprès du dieu solaire ${ }^{42}$. Aux valeurs de génération et de production du monde naturel s'opposent désormais les principes de permanence et d'identité du moi, qui poussent l'homme à nier la relation vivante qui le lie au cosmos (la terre devient un caveau), puis aux autres (les autres deviennent des ennemis ou des ombres) et enfin à lui-même (scindé en un corps périssable soumis aux plaisirs et une âme éternelle qui attend le jugement).

Hétérodoxie et Orthodoxie en Philosophies

17 La double polarité Nord/Sud et Masculin/Féminin qui traverse l'histoire informe au plus profond également la pensée : elle renvoie à une division interne à la Pensée entre pensées dominantes et pensées hétérodoxes, propre à toutes les cultures.

18 Ainsi, en Chine, l'opposition entre le taoïsme mystique et le confucianisme normatif dominant se marque, comme par hasard, par une telle différence géographique d'ancrage :

19 «Le Huainan zi fut écrit dans le Sud de la Chine, en une région qui fut profondément influencé par la culture de Chu, assez différentes de la culture de la capitale, située dans le Nord. C'est, si l'on veut, l'opposition pérenne entre, d'une part, la culture du fleuve Jaune, plus classique et traditionnelle, d'orientation pratique, centrée sur le confucianisme et le légisme, et d'autre part la culture du Yangzi, plus idéaliste, novatrice, et favorable aux enseignements des cent écoles, baijia, mais avant tout à ceux du taoïsme ${ }^{43}$.»

20 Le fait qu'un tel rapport géographique au Sud aille de pair avec un mode de penser hétérodoxe se retrouve dans le monde occidental. En effet, par rapport à la position centrale de l'Athènes platonicienne, l'Élée de Parménide se situe au Nord-Ouest et la Macédoine d'Aristote au Nord-Est tandis que l'Alexandrie de Plotin se situe au SudOuest et la Milet d'Héraclite se situe au Sud-Est - Proclus fut le premier à le noter ${ }^{44}$.

21 À l'époque moderne, la confrontation de Giordano Bruno (de Nola, dans la province de Naples) et des docteurs d'Oxford, relatée dans le Banquet des Cendres, illustre la même différence. À bien des égards, le bûcher de Bruno et l'emprisonnement de Campanella, commandés par l'orthodoxie religieuse, ont brisé la possible naissance d'une philosophie à la fois naturaliste et néoplatonicienne en Italie, privant sans doute en Europe la pensée du pôle Sud de sa mentalitée ${ }^{45}$.

22 Ailleurs, en Orient à nouveau, en Inde, sur le plan de la différence masculin/féminin, une des particularités du tantrisme, hétérodoxe par rapport à l'orthodoxie brahmanique, est la place privilégiée, tant au point de vue métaphysique ${ }^{46}$ que pratique $^{47}$, accordée à la femme :

23 «Le développement tantrique a favorisé le retour au pouvoir, dans l'hindouisme populaire, de la Déesse Mère... [...] Il est parfaitement possible que, dans cette réinstauration de la Déesse, tant parmi les cultes populaires que dans la profonde philosophie du Tantra, nous ayons un autre signe de la reviviscence de la tradition matriarcale, non aryenne, préaryenne, propre aux époques dravidiennes ${ }^{48}$. "

24 En Occident philosophique, ce lien entre pensée du sensible et symbolique féminine se retrouve exemplairement chez Schelling, dans le splendide Clara, où l'héroïne éponyme clame l'union du corps et de l'esprit dans une âme de vie placée au cœur de la nature : 
«La corporéité n'est pas l'imperfection, au contraire : lorsque le corps est pénétré par l'âme, il est la plénitude de la perfection. La seule vie de l'esprit ne suffit pas à notre cœur [...] il m'est à nouveau apparu de façon tout à fait claire et intime, cela m'a touchée au cœur, que nous sommes des enfants de la nature, que nous lui appartenons, selon notre première naissance, et ne pouvons jamais nous séparer d'elle...49 "

Un exemple : le monde médiéval méridional et sa disparition

Cette hypothèse des différents niveaux religieux et philosophiques de l'expression d'une différence entre pensée «masculine » du Nord/Ouest et pensée «féminine » du Sud/Est aurait peu d'intérêt si l'on ne pouvait en suivre l'incarnation plutôt précise et répétée dans l'histoire.

Ainsi, l'un des exemples les plus significatifs du processus de double négation du principe féminin et de la polarité Sud dans l'histoire du déploiement historique des forces naturelles de vie captée par l'appareil psychique humain se trouve dans l'histoire de France. L'opposition entre Nord et Sud y est d'abord une réalité géographique, datant du limes romain et séparant le monde barbare et le monde civilisé par une ligne allant approximativement de Lyon à Bordeaux. Le point de vue du Nord se reflète dans ces propos de Renan :

"Je ne sais si je me trompe, [...] mais il y a une vue d'ethnographie historique qui s'impose de plus en plus à mon esprit. La similitude de la France du Nord et de l'Angleterre m'apparaît chaque jour davantage. Notre étourderie vient du Midi et, si la France n'avait pas entraîné le Languedoc et la Provence dans son cercle d'activité, nous serions actifs, protestants, parlementaires ${ }^{50}$.»

À ceci répondent les propos de Mary-Lafon quand il oppose "aux Méridionaux du Moyen Âge, raffinés, amoureux de liberté, la "brutale barbarie" des "chevaliers d'outreLoire", pillards, violents, fanatiques ${ }^{51} »$. Une telle opposition entre pensée du Nord et du Sud et réalité masculine et féminine prend forme concrète au cours du douzième siècle. Elle se manifeste, au cœur du pouvoir, par la répudiation d'Aliénor d'Aquitaine, protectrice des troubadours occitans, par Louis VII ${ }^{52}$. Du point de vue d'une histoire symbolique, il est remarquable que, si cette répudiation du principe sud/féminin en la personne d'Aliénor a eu pour conséquence le commencement de la guerre de Cent Ans, cette même guerre ne s'est achevée que par le secours d'une autre femme, mais cette fois-ci venue du Nord, qui faisait honneur non plus à la plume mais à l'épée, à savoir Jeanne d'Arc (l'élément féminin n'étant donc réintégré qu'en se fondant dans le monde masculin de la guerre - de même que si la femme aujourd'hui accède au monde de l'homme, c'est sans changer radicalement l'aspect masculin de ce monde). Un dernier événement à cette époque consacra la domination définitive du Nord sur le Sud : ce fut la croisade des Albigeois qui détruisit un pan de la culture méridionale :

« Deux cents ans, il [le mouvement communal] valut aux villes de l'île-de-France, de la Picardie, de la Champagne, une civilisation touffue, confuse d'apparences, mais d'un rythme intérieur puissant, qui contraignit la féodalité à se réfugier dans les campagnes pour y provoquer la Jacquerie deux ou trois siècles plus tard et à se ruer, sous prétexte d'exterminer l'hérésie, sur les cités méridionales dont elle écrasa la culture et le libre esprit grandissant. Ce fut la rançon terrible de la liberté du Nord $^{53}$."

Que l'écrasement de la mystique cathare ait eu un prétexte intéressé n'échappa pas aux représentants de ce monde culturel détruit, à savoir aux troubadours eux-mêmes : « les Français n'ont de pitié que s'ils en tirent quelque profit: je ne vois point chez eux 
d'autre loi [...] Ils vivent dans l'abondance et le bien-être, et leur religion n'est que tromperie et trahison ${ }^{54}$. Or, on sait que la poétique des troubadours fut le lieu de naissance de l'amour courtois, inversant les rapports de domination homme/femme à travers la loi nouvelle de l'Amour ${ }^{55}$. Ainsi, répudiation d'Aliénor, croisade des Albigeois, disparition de la culture courtoise des troubadours réalisent en un siècle en France la répétition d'un mouvement plus général, géographique, symbolique et historique de double négation de la vie en son aspect sud/féminin (les effets aujourd'hui de cette double négation sont visibles dans la traite mondiale des ressortissantes des pays du Sud-Est $\left.{ }^{56}\right)$.

Conclusion

Plutôt que de penser l'histoire du monde en termes de progrès, nous avons essayé d'en rendre compte à partir d'un renversement de valeurs par lequel la polarité Sud/Est fondée sur la Passion et la Contemplation s'est effacée devant la polarité Nord/Ouest fondée sur la Raison et l'Action. Le principe d'une géographie symbolique de la morale consiste à affirmer que cette domination du principe Nord/masculin sur celui Sud/ féminin est comme la conséquence et le symptôme du renversement des valeurs faisant passer du sacre de la vie au culte de la mort.

Bien entendu, c'est là un canevas très large demandant d'infinies rectifications.

La première étant que ce renversement est lui-même en sa phase finale de réalisation et qu'un mouvement global de retournement de ce renversement semble se mettre en place: le surgissement du féminisme au début siècle dernier et le rattrapage économique des pays asiatiques au début de ce siècle semblent indiquer qu'un nouvel équilibrage des forces se fait.

De plus, même si nous essayons le plus possible de nous placer du point de vue de l'histoire, ce qui compte c'est peut-être moins le fait que le renversement ce soit produit à tel ou tel moment pour telle ou telle cause, que le fait qu'il renvoie à l'existence d'une telle bipolarité présente au sein de l'être et que l'histoire ne fait qu'actualiser.

Plus encore, il faudrait préciser que nous nous plaçons au niveau non empirique de la différence interne: le terme Sud ne désigne ni un pays ni un hémisphère, car la géographie est fractale (où que l'on soit, il y a toujours un Nord, Sud, un Est et un Ouest - pour le Londonien, l'Italie, c'est le Sud mais pour le Milanais, le Sud, c'est Naples); quant à l'adjectif féminin, il ne renvoie pas à une identité sexuelle mais à un aspect sexué - étant dit que le pôle féminin et le pôle masculin coexistent et prédominent plus ou moins chez la femme comme chez l'homme.

Ce qui importe pour nous, c'est cette idée d'un potentiel de vie qui s'incarne à travers l'histoire selon deux directions privilégiés : masculin/nord/ouest ; féminin/sud/est. La polarité féminin/sud/est serait le symbole de tout ce qui dans la vie favorise la vie, entendue comme puissance de production de différences tandis que la polarité masculin/nord/ouest serait le symbole de tout ce qui dans la vie favorise la mort, entendue comme ce qui veut maintenir l'identité à soi et préfère l'autodestruction à la perte de soi. 


\section{NOTES}

1. Cf. Gérard de Nerval, Les Chimères, « Myrtho », Euvres, p. 696, Garnier, 1966.

2. Cf. Friedrich Nietzsche, Écrits Posthumes, 1870-1873, Gallimard, 1975, « La philosophie à l'époque tragique des grecs ", traduction Michel Haar, Marc B. de Launay, p. 248 ; cf. aussi Friedrich Nietzsche, Les Philosophes préplatoniciens, § 10 Xénophane et Parménide, texte établi par Paolo D'Iorio, traduction Nathalie Ferrand, éditions de l'éclat, 1994, p. 242, p. 244.

3. Cf. Friedrich Nietzsche, Généalogie de la morale, Deuxième Traité, traduction E. Blondel, $\mathrm{O}$. Hansen-Love, T. Leydenbach, P. Pénisson, Flammarion, 1996. D'un point de vue très concret, ce rapport de la religion à la mort se reflète dans l'appropriation de l'espace de la mort par l'autorité religieuse ; cf. Régis Bertrand, Rives, n 6, 2000 : «Les évêques sont, sous l'Ancien Régime, seuls compétents en droit pour affecter à la sépulture un lieu de culte qui n'en renferme pas encore, de même que pour décider du principe de la création ou la désaffectation d'un cimetière. »

4. Cf. Georges Duby, «L'héritage », pp. 193-216 [212-213]. Cf. Christian Grateloup, « Les espaces de la mondialisation, héritages et dynamiques », pp. 132-143, Qu'est-ce que la société ?, Odile Jacob, 2000, p. 138 : « Les images que nous avons en tête du Monde sont des planisphères. Cette figure nous semble neutre [...] Or le choix de placer au nord l'“orientation" (terme qui littéralement signifie tourné vers l'est), et non vers le sud, comme les cartes chinoises anciennes, est une conséquence de la position assez septentrionale de l'Europe et son écriture à partir du haut. » 5. Cf. Gabriel Camps, Introduction à la préhistoire, Librairie Académique Perrin, 1982.

6. Cf. Alain Begueme, «Aux origines de la guerre : actes de violence et massacres dans le néolithique européen ", Revue des Études Anciennes, 2001, tome 103, n³-4, p. 329-342. Bien entendu la guerre primitive existe mais sous une forme particulière, cf. Pierre Clastres, Archéologie de la violence, Aube, 2005.

7. Cf. Gordon Childe, Le mouvement de l'histoire, Arthaud, 1961, chapitre VIII. Colin Renfrew s'est opposé à la thèse de Childe en proposant à la place d'une irruption guerrière une émigration lente et pacifique liée à l'émergence des pratiques agricoles (L'Énigme indo-européenne. Archéologie et langage, Flammarion, Paris, 1990). La thèse de Childe a été renouvelée par Marija Gimbutas qui, en s'appuyant sur les fouilles pratiquées entre la Dniepr et la Volga révélant de nombreuses sépultures individuelles d'hommes entourés d'armes et de restes de chevaux, a émis l'hypothèse d'une « civilisation des kourganes ", développée à partir du milieu du cinquième millénaire et se mettant en mouvement à partir du milieu du quatrième en trois vagues successives (notons qu'autour de cette même région des fouilles récentes ont mis en évidence des villages épipaléolithiques déjà bien structurés : cf. Stéphane Péan, Ninelj Kornietz et Dimitrii Nuzhnyi, «Vivre du mammouth au Paléolithique et Ukraine », Pour la Science, avril-juin 2004, dossier $n^{\circ} 43$, pp. 82-89); d'autre part, un problème de chronologie se pose : la thèse de Gimbutas ne tient que dans le cas d'une chronologie basse, remise en question, cf. N. Kazanas, «Indigenous Indo-Aryans and the Rigveda ", Journal of Indo-European studies, 2002, vol. 30, n 3-4, pp. 275-334.

8. Cf. Thomas Gamkrelidze et Vjacheslav Ivanov, «L'aube des langues indo-européennes », Pour la Science, octobre 1997, dossier $n^{\circ} 17$, pp. 54-60.

9. Cf. Gérard Fussman, « Entre fantasme, science et politique. L'entrée des Aryas en Inde », Annales (Histoire, Science sociale), juillet-août, 2003, n 4, pp. 778-814.

10. Cf. Iaroslav Lebedynsky, "Les nomades d'Eurasie : une "civilisation" des steppes », Pour la Science, $\mathrm{n}^{\circ} 337$, novembre 2005, pp. 58-63 : «À la charnière de l'âge du bronze et de l'âge du fer, 
vers le début du Irr $^{\text {er }}$ illénaire avant notre ère, des populations d'éleveurs et d'agriculteurs des steppes eurasiatiques ont abandonné leurs villages [...] et se sont spécialisées dans un élevage nomade extensif. »

11. Cf. F. Brunet, «Asie centrale : vers une redéfinition des complexes culturels de la fin du Pléistocène et des débuts de l'Holocène ", Paléorient, 2002, nº 28/2, pp. 9-24.

12. Cf. Maurice Godelier, « Anthropologie économique », Encyclopcedia Universalis, 2003.

13. Cf. A. Moret et G. Davy, Des clans aux empires, L'organisation sociale chez les primitifs et dans l'Orient ancien, 1923, La Renaissance du Livre, Annexe, pp. 427-8.

14. Cf. Roland Martin, L'Art Grec, Chapitre premier, Librairie Générale Française, 1994 ; cf. Mashkan-shapir était-elle une cité égalitaire ? ", Pour la Science, pp. 20-23, Dossier n 25, octobre 1999.

15. Cf. Peter Warren, « Les palais minoens », Pour la Science, pp. 36-42, Dossier $n^{\circ} 25$, octobre 1999. Cf. Henri-Paul Francfort et Olivier Lecomte, «Irrigation et société en Asie Centrale des origines à l'époque achéménide », Annales mai-juin, 2002, n³ 3, pp. 625-663.

16. Cf. Georges Dumézil, L'Idéologie tripartite des indo-européens, 1958. Pour un état plus récent de la question, voir les articles de la Revue de l'Histoire des Religions, vol. 2, 1991, « Histoire des religions et comparatisme : la question indo-européenne ». Cf. Dominique Briquel, « Le règne de Tullus Hostilius et l'idéologie indo-européenne des trois fonctions ", Revue de l'Histoire des Religions, vol. 1, 1997 ; Emily Lyle, « Which Triad ? A Critique and Development of Dumézil's Tripartite Structure". Revue de l'Histoire des Religions, vol. 1, 2004.

17. Cf. Georges Dumézil, L'Idéologie tripartite des indo-européens, 1958, III Les théologies tripartites, in Mythes et Dieux Indoeuropéens, Flammarion, 1992, p. 146. Dumézil sera encore plus net dans sa préface à la dernière édition de Heur et malheur du guerrier : on ne peut réunir d'aucun trait indoeuropéen commun les divers traits propres à la troisième fonction ; tous les thèmes qui s'y rattachent relèvent une étroite liaison à une base géographique, topographique et ethnique définie, c'est-à-dire indigène.

18. Cf. Tite Live, Histoire Romaine, Livre I, § 9, traduction Annette Flobert, Flammarion, 1995, pp. 68-71.

19. Cf. Emmanuel Dupraz, "Sur la différence des sexes dans le De Raptu Proserpinae », Revue des études anciennes, 2003, vol. 105, n 1, pp. 251-266 : «Le De Raptu Proserpinae de Claudien se caractérise par une composition très précise qui met en scène deux couples : Cérès et Pluton, Nature et Jupiter. Dans chacun de ces couples, une opposition à la fois métaphysique et psychosexuelle est à l'oeuvre: la femme est mère et positivité, l'homme est père et destruction. Ces oppositions et ces complémentarités permettent de faire du rapt de Proserpine un symbole en même temps qu'un épisode de l'Histoire de l'humanité.»

20. Cf. Luce Irigaray, Entre Orient et Occident, Grasset, 1998 : « Les nomades conquièrent leur territoire contre la familiarité du premier site, contre les sédentaires, contre les valeurs plus maternelles, plus féminines. Ils créent une culture de l'entre-hommes, ennemis ou complices, dont la divinité est plutôt patriarcale, le Dieu-Père, partout présent et jamais là, que l'on suit, qui vous accompagne, qui vous fustige et vous assiste, qui impose ses lois à des peuples itinérants échappant aux mesures d'une vie plus naturelle. »Cependant, cette affirmation ne semble pas entièrement juste : le mythe des Amazones atteste à la fois du rôle, avéré, que peuvent jouer les femmes en milieu « nomadisant » où elles peuvent participer aux activités guerrières et régner sur un clan et du scandale que cela représentait pour l'homme sédentaire.

21. Cf. Johann Jakob Bachofen, Das Mutterrecht, Stuttgart, 1861. Cf. Bernard Valade, « J.J. Bachofen », Encyclopcedia Universalis, 2003 : « Une lettre (G.W., X, no 96), adressée le 23 octobre 1857 par Bachofen à A. Gervasio, montre que c'est à l'étude de la famille comme institution 
sociale que l'ont finalement conduit ses recherches sur l'Antiquité classique : "Quoi de plus surprenant, écrit-il à l'archéologue italien, que de voir la femme des premiers temps de l'histoire humaine occuper le rang et la position qu'un développement plus avancé a irrévocablement assignés aux êtres du sexe masculin. En rassemblant les débris épars de cette institution (la gynécocratie des peuples de l'Antiquité), je suis arrivé au résultat que ce système n'est pas un phénomène isolé mais la propriété de toute l'humanité." » Cet aspect premier et archaïque du monde matrilinéaire semble plutôt confirmé - cf. par exemple, Jean A. Trejaut, Toomas Kivisild and al., "Traces of Archaic Mitochondrial Lineages Persist in Austronesian-Speaking Formosan Populations”, PLoS Biology, Volume 3, Issue 8, August 2005 : “the proto-Austronesian communities were matriarchal and matrilocal (as the Amis tribe still is in Taiwan)...".

22. Cf. Jean Varenne, « Apsara », Encyclopcedia Universalis, 2003 : « [Le] panthéon védique [est] organisé selon trois niveaux : au sommet, les grands dieux, souverains du domaine cosmique [...]; en dessous d'eux, des dieux secondaires qui assistent les précédents dans leurs fonctions [...]; plus bas enfin se situent les génies du monde intermédiaire [...] Parmi ces "génies" prennent place les Apsaras, qui correspondent à la fois aux nymphes de la mythologie grecque et aux Walkyries des légendes germaniques. Ce sont, en effet, des femmes (toujours jeunes et belles) qui volent dans l'atmosphère, mais fréquentent volontiers les lacs et les rivières [...] Les théologiens mettent en garde contre les Apsaras ceux qui veulent progresser spirituellement : elles apparaissent alors comme les tentatrices par excellence. Dans l'hindouisme classique, ces fées deviennent franchement démoniaques - du moins dans les textes doctrinaux -, les contes, le folklore, la religion populaire continuant au contraire à les vénérer et à solliciter leurs faveurs. » 23. Cf. Catherine Basset, Musique de Bali à Java, pp. 108-09, Cité de la Musique/Actes Sud, 1995.

24. Cf. Michelet, La Sorcière, Flammarion, 1966. Cf. Maurice Aymard, « Espaces », pp. 191-223, in Fernand Braudel, La Méditerranée I L'Espace et l'Histoire, Flammarion, 1985 : « Maîtresse du cycle de la naissance et de la mort, la femme entretient un rapport privilégié avec les puissances souterraines ».

25. Cf. Cicéron, De finibus, V, 49, traduction F. Buffière, in Les mythes d'Homère et la pensée grecque, p. 385 : «Ce n'est, apparemment, ni la douceur de leur voix, ni la nouveauté ou la variété de leurs chants qui savaient retenir au passage les navigateurs, mais l'assurance qu'elles donnaient de savoir bien des choses... Homère a bien vu quelle serait l'invraisemblance de sa fable, s'il suffisait de simples chansonnettes pour emprisonner dans les mailles du filet un homme comme Ulysse : c'est donc la science que promettent les Sirènes, la science, dont on comprendrait fort bien qu'elle contrebalançât le désir de la patrie, dans un cœur épris de sagesse ».

26. Cf. Fernand Braudel, « L'histoire », pp. 157-190, in Fernand Braudel (sous la direction de), La Méditerranée I L'Espace et l'Histoire, Flammarion, 1985 : «Le processus qui menace la Méditerranée et qui aura finalement raison d'elle, ce n'est rien moins que le déplacement du centre du monde, de la mer Intérieure à l'océan Atlantique ».

27. Cf. Jacques Cauvin, Naissance des divinités, naissance de l'agriculture, CNRS, 1994.

28. Cf. Jean-Louis Brunaux, «La religion celtique : une origine orientale? », Pour la science, $\mathrm{n}^{\circ} 309$, pp. 24-29, juillet 2003 : « Les Celtes anciens comme la plupart des Scythes et de nombreux peuples du Proche-Orient, étaient avant tout des nomades... Il y a deux catégories de dieux celtes : les Ouraniens (célestes) et les chtoniens (souterrains)... Plusieurs auteurs antiques rapportent que les Gaulois croyaient en l'immortalité de l'âme selon une théorie proche de la métempsychose... Une personne ayant eu une vie exemplaire, conforme à la morale édictée par les druides, pouvait se voir récompenser par un séjour éternel auprès des dieux. Aspect central de la civilisation celtique, leurs pratiques guerrières illustrent l'importance que les Gaulois accordaient au mérite pour le salut de leur âme.» 
29. Cf. A. Moret et G. Davy, Des clans aux empires, L'organisation sociale chez les primitifs et dans l'Orient ancien, 1923, La Renaissance du Livre, p. 210.

30. Cf. Le Livre des Morts des Anciens Égyptiens, Dervy-Livres, 1991, traduction Grégoire Kolpaktchy, Chapitre LXXXIII, p. 163. « Voici que je plonge dans la Matière Primordiale /Et que je deviens Khepra, dieu des Métamorphoses. /Je bourgeonne par la force universelle de bourgeonnement ... /En vérité je porte en moi / Les germes et les possibilités de tous les dieux. »

31. Cf. Le Livre des Morts des Anciens Égyptiens, idem, Chapitre CXC, p. 314. « Je suis Toth maitre des deux cornes de la Lune ; /mon écriture est parfaite et mes mains sont pures. /Je déteste le Mal et j'abhorre l'Iniquité ; /Je fixe par écrit la Justice divine.../ Voici que Râ se couche à l'Horizon/ Pareil à Osiris... »

32. Cf. Le Livre des Morts des Anciens Égyptiens, idem, Ch. LXIV, p. 134, Ch. LXVIII, p. 142 : « ... tout être ressuscité/ Pendant qu'il passe dans le Royaume des Morts,/ Par des transformations successives,/ Péniblement cherche son chemin/ À travers la Région des Ténèbres "; « Voici que les Portes du Ciel me sont ouvertes/ Et que les Portes de la Terre ne s'opposent plus à mon passage ".

33. Cf. A. Grenier, La Religion Étrusque et Romaine, P.U.F., 1948.

34. Cf. Otto Rank, Don Juan et Le Double Essais psychanalytiques, I Le Double, Payot, traduction S. Lautman, 1973, 4. L'Ombre, représentation de l'âme : « Rochholz, qui s'est particulièrement occupé de la croyance à l'ange gardien, dit que la signification primitive de cette croyance était favorable mais s'est transformée plus tard, quand la croyance en l'au-delà a pris racine, en une signification défavorable : la mort. Ainsi, l'ombre de l'homme qui, auparavant, a été un ange gardien prêt à lui porter secours dans la vie, doit se transformer en un spectre effroyable qui persécute et martyrise l'homme jusque dans la mort », 7. La croyance à l'immortalité du moi : « Nos recherches semblent montrer qu'au début ce Double était l'âme de l'homme vivant et que peu à peu il est devenu l'âme du mort.»

35. Cf. Homère, Iliade, XIV, v. 201 : « ... Okéanos, origine des dieux... », v. 246 : « ... le fleuve Okéanos, cette source de toutes choses... » - traduction Leconte de Lisle. Cf. aussi Mahā Nārāyaöa Upanishad, traduction Jean Varenne, De Boccard, 1988, p. 77, §§ 312-316 : « Les eaux en vérité sont cet univers tout entier ; les eaux sont tous les êtres [...] les eaux sont l'ensemble des divinités. » 36. Cf. Hésiode, Théogonie, v. 447, traduction Annie Bonnafé, Rivages, 1993, p. 99 : « et Zeus, maître de l'idée, père des dieux et des hommes... »; v. 529, p. 107 : «Zeus, l'Olympien souverain des hauteurs...»

37. Cf. Giorgio Colli, La Sagesse grecque, Volume 1, éditions de l'éclat, 1990, Pseudo-ératosthène, 24, Orphée, 4 [B 2], p. 199, traduction Marie José Tramuta. Ce qui chez Héraclite était opposition de contraires (« Le même sont Hadès et Dionysos... », cf. Héraclite, B XV, traduction Jean-Paul Dumont, Les Présocratiques, p. 150, Gallimard, 1988) devient identité forcée.

38. Cf. Louis Gernet, "Dionysos et la religion dionysiaque ", in Anthropologie de la Grèce antique, Flammarion, 1982. Cf. Paul Vicaure, «Place et figure de Dionysos dans la tragédie de Sophocle », Revue des Études Grecques, 1968, t. CXXXI, pp. 351-373. Cf. Isabelle Tassignon, « Les éléments anatoliens du mythe et de la personnalité de Dionysos ", Revue de l'Histoire des Religions, $n^{\circ}$ 3, 2001. 39. Cf. Euripide, Les Bacchantes, 704-711, traduction Marie José Tramuta : « Se saisissant de son thyrse, une bacchante heurte un rocher,/ d'où jaillit une limpide coulée d'eau ; / une autre frappe la terre de sa férule,/et le dieu pour elle fait surgir une source de vin ; /enfin celles qui sont saisies du désir du blanc breuvage /grattaient le sol de leurs ongles/ et en recueillaient du lait en abondance : mais des thyrses de lierre/ s'écoulaient de doux ruisseaux de miel. ». 
40. Cf. Giorgio Colli, La Sagesse grecque, Volume 1, éditions de l'éclat, 1990, Dionysos, A 5, p. 57, Euripide, Les Bacchantes, 1019-1020, traduction Marie José Tramuta : « Viens, ô Bacchos, de ton visage qui rit jette un lacs / mortel autour du chasseurs des bacchantes... »

41. Cf. idem, Éleusis, 3 [B 1], p. 111, Philodème, 31, 35-6 : «Évohé, ô Iobacchos, ô Péan.../ Tu as ouvert aux mortels/ un lieu à l'abri des souffrances, un havre exempt de douleur. »

42. Cf. idem, Orphée, 4 [A 5], p. 121, Pindare, Fr. 137 : « Et ceux dont Perséphone agréera la punition/pour le deuil ancien, à la neuvième année elle renvoie de nouveau/les âmes à la splendeur du soleil d'en haut. »

43. Cf. Philosophes taoïstes II. Huainan zi, sous la direction de Charles Le Blanc et Rémi Mathieu, Introduction générale, p. XIX, Gallimard, 2003. Cf. aussi Léon Wiegler, Histoire des croyances religieuses et des opinions philosophiques en Chine depuis l'origine jusqu'à nos jours, Dix septième leçon, p. 148, Hien-hien, 1922 : « À noter aussi, que le premier développement du Taoïsme se fit dans les provinces méridionales de la Chine.»

44. Cf. Proclus, Commentaire sur le Parménide de Platon, 659, 250, $\mathrm{II}^{\mathrm{e}}$ partie, traduction d'A. E. Chaignet, Paris, 1900, p. 94 : «L'Ionie sera donc le symbole de la nature, l'Italie de la substance intellectuelle : Athènes le symbole de la substance moyenne par laquelle les âmes réveillées remontent de la nature à la raison ».

45. Cf. Luca Salza, Métamorphose de la Physis. Giordano Bruno, Vrin, 2005.

46. Cf. Kaulajnananirnaya of Matsyendranath, Prachya Prakashan, 1986, traduction par Michael Magee, Patala 23: "Worship carefully a woman or a maiden as she is Śakti, sheltered by the Kulas." Cf. André Padoux, « Tantrisme », Encyclopcedia Universalis, 2003 : « Caractéristique du tantrisme est sa conception de la divinité [...] La création est l'œuvre de cette énergie féminine : elle en est toute pénétrée et se déploie comme un vaste jeu cosmique. » Cf. aussi Mahā Nārāyaöa Upanishad, traduction Jean Varenne, De Boccard, 1988, p. 83, § 334 : «Ô déesse tu es l'énergie, tu es la forme, tu es la puissance, tu es l'éclat [...] Tu es l'universelle vie de toutes choses, la vie de l'univers. » 47. Cf. Tantra Yoga. Le Vij-ânabhaïrava tantra, traduit et commenté par Daniel Odier, pp. 43-44, Albin Michel, 2005 : « D'après la tradition, l'école Pratyabhija s'est développée avant le début du Kaliyuga, ou âge sombre qui a commencé environ 3200 ans avant notre ère. Shiva, voyant l'avènement de l'âge obscur, décida de raviver le shivaïsme en le transmettant, sur le mont Kailasha, au sage Durvasa. Il lui exposa les tantra en lui demandant de les transmettre à son tour. Durvasa eut un premier fils qu'il ne jugea pas digne de recevoir les enseignements ultimes, il lui transmit donc les tantra dualistes. Plus tard, il eut un autre fils auquel il transmit les tantra qui sont à mi-chemin entre le dualisme et le non-dualisme. Enfin, il eut une fille, Ardhatryambaka, qui seule se montra capable de comprendre les soixante-quatre Bhaïrava tantra suprême de l'école non duelle [...] De là date l'importance capitale des maîtres femmes dans le shivaïsme, car elles peuvent, dit-on, réaliser les enseignements trois-cent-soixante-cinq fois plus rapidement qu'un homme [...] De cette présence si forte des yoginî vient aussi l'ouverture des tantra dont les enseignements s'adressent à tous, sans aucune restriction de sexe, de caste ou de race. "

48. Cf. Heinrich Zimmer, Les philosophies de l'Inde, Troisième partie, Chapitre V, pp. 446-7, traduction Marie-Simone Renou, Payot, 1997.

49. Cf. F. W. Schelling, Clara ou Du lien de la nature au monde des esprits, traduction Élisabeth Kessler, L'Herne, 1984, pp. 166-7.

50. Cité par Fernand Braudel, L'Identité de la France I, Espace et Histoire, Arthaud, 1986, p. 75.

51. Cité par Fernand Braudel, ibidem, p. 77.

52. Cf. Jean Favier, « Aliénor d'Aquitaine », Encyclopcedia Universalis, 2003 : « Fille de Guillaume X, duc d'Aquitaine, [Aliénor d'Aquitaine] fut mariée dès son avènement au prince Louis, qui montait, deux semaines plus tard, sur le trône de France sous le nom de Louis VII [...] L'union 
était mal assortie, la légèreté et la sensualité d'Aliénor s'accordant mal avec la dévotion ascétique du roi."

53. Cf. Élie Faure, Histoire de l'art, L'Art Médiéval, Le Livre de Poche, 1964, p. 240. Sur le plan économique, la domination actuelle du Nord sur le Sud est consommée par le déplacement, au $\mathrm{XVI}^{\mathrm{e}}$, siècle, du centre économique mondial de Venise à Anvers et de Gênes à Londres - à peu près au moment où la Réforme brise la Renaissance. Cf. Fernand Braudel, La Méditerranée I L'espace et l'histoire, Flammarion, 1985, «L'Histoire », pp. 177-183 ; cf. Fernand Braudel / Georges Duby, La Méditerranée II Les hommes et l'héritage, « Venise », pp.176-181, Flammarion, 1986.

54. Cf. Bernard Sicart de Marvejols, sirventès [poème en forme de diatribe] «Ab grèu cossire », in Anthologie des troubadours, Textes choisis, présentés et traduits par Pierre Bec, Union Générale d'Éditions, 1979, p. 281. Cf. aussi Pèire Cardenal, sirventès « Tartarassa ni voutor », ibidem, p. 309 : «Le milan ni le vautour ne flairent plus vite la charogne que les prédicateurs le séjour du riche [...] Les Français et les clercs font la louange du mal, car cela leur procure des avantages... » 55. Cf. Cercamon, "Quant l'aura doussa s'amarzis ", in Anthologie des troubadours, Textes choisis, présentés et traduits par Pierre Bec, Union Générale d'Éditions, 1979, pp. 100-1. « Lorsque le monde entier se couvre de ténèbres, tout resplendit là où elle se trouve. » Ajoutons que cet hommage à la Dame, avec la mort des troubadours du Sud et l'avènement des trouvères du Nord se transforma en culte christianisé de Marie. Mais c'est déjà vrai dans le passage du douzième au treizième siècle : cf. Folquet de Lunel, « Cansó à la Vierge », idem, p. 317-322 : «Chanson à la Vierge qui reprend systématiquement, les déviant de leur fonction première, tous les topiques de la poésie amoureuse ».

56. Cf. Richard Poulin, «Le temps de la vénalité sexuelle », Actuel Marx, n² 28, novembre 2004 : « La traite des femmes et des enfants de l'Asie du Sud et de l'Asie du Sud-Est est estimée à 400000 personnes par année [...] D'après l'ONU, au cours des trente dernières années, en Asie seulement, la traite de femmes et d'enfants aux fins "d'exploitation sexuelle" a fait plus de 30 millions de victimes. »

\section{RÉSUMÉS}

Le but de cet article sera de proposer une relecture, voire une réécriture de la Généalogie de la morale à partir des nouvelles données de l'histoire comparée des religions en même temps qu'à travers l'optique contemporaine des recherches sur les différences hommes-femmes ("Gender Studies »). Chez Nietzsche, «la morale » désigne un système global de faits et d'idées, de pensées et d'attitude déterminé par la volonté de s'opposer à ce qu'il y a de vital ou créateur dans la volonté de puissance, dans les forces qui traversent l'homme. La morale, c'est ce qui, dans la vie, est retourné contre la vie, c'est ce qui utilise la force propre à la vie (expansion, transformation, renouvellement) pour étouffer l'émergence de toutes possibilités nouvelles d'existence. Il s'agira de montrer ici comment la «morale » se constitue par un processus de retournement des valeurs de «vie » en celles de "mort » - un tel renversement des valeurs étant lié à des facteurs de polarisation géographique (nord/sud, ouest/est) et sexuelle (masculin/féminin) des forces. Par là, il s'agit au fond de proposer un nouveau paradigme pour penser le devenir historique, opposé à la pensée d'un universel abstrait s'accomplissant progressivement dans l'Histoire. Les idées ont 
un sexe et un site : ni elles ne sont éternelles ni elles ne "progressent » au cours de l'histoire ; elles se transforment et varient selon les lieux et les âges.

The purpose of this paper is to suggest a re-reading, even a re-writing of the Genealogy of Morals based on the new data derived from the comparative history of religions, and through the contemporary perspective of "Gender studies". For Nietzsche, "morals" refers to a whole system of facts and ideas, thoughts and attitudes determined by the will to counter what is vital or creative in the will-to-power, in the forces flowing through man. Morals is that part of life which is turned against life itself, using the intrinsic force of life (expansion, transformation, regeneration) to stifle the emergence of all new possibilities of existence. We would like to show here how "morals" is formed through a process of reversing the values of "life" into the values of "death"; such a reversal being related to the way forces are polarized geographically (north/ south, west/east) and sexually (masculine/feminine). Thus, we will be suggesting a new model to reflect on historical becoming, as opposed to an abstract universality gradually achieving itself as History. Ideas have a sex and a location: they are neither eternal, nor "progressing" in the course of history; they transform and vary according to places and times.

\section{AUTEUR}

\section{JEAN-YVES HEURTEBISE}

Jean-Yves Heurtebise est docteur de philosophie, rédacteur associé à la revue Sens Public et président de l'association RezoDoc des jeunes chercheurs d'Aix-Marseille Université. Ses travaux portent sur des domaines divers : esthétique (direction d'un colloque sur le « cinéma politique » au Collège International de Philosophie, à paraître ; interventions aux Ecrans philosophiques de Montreuil sur Wong Kar Wai ; «Pratiques de la danse et Catégories du corps », Les Amandiers, à paraître), philosophie des sciences (direction du colloque « la vie et le temps » avec Pierre Gilles de Gennes et Dominique Lecourt, Sens Public, février 2008) et histoire de la philosophie (La vie comme liaison, livre en préparation ; "Nietzsche : l'Éternel retour et la philosophie du temps », article en soumission). 